\title{
THERMAL IMAGING MICROSCOPE AND APPLICATIONS
}

\author{
D. U. Kim, C. B. Jeong, W. J. Choi, S. Y. Ryu, K. S. Chang* \\ Division of Scientific Instrumentation, Korea Basic Science Institute \\ *Presenting and Corresponding Author: ksc@kbsi.re.kr
}

We demonstrate quantitative micro-thermography systems, including infrared thermal microscopy and thermoreflectance microscopy. Several applications of micro-thermography, such as quantitative measurement of the surface and sub-surface temperature distribution of semiconductor devices, hot spot detection for failure analysis of semiconductor integrated circuits, and detection of defects in optical materials will be presented.

KEYWORDS: Infrared, Thermoreflectance, Photothermal, Microscopy

More powerful and faster micro-electronic devices mean hotter devices, which can lead to a decrease in performance and lifetime. Thus, thermal analysis under their operating conditions become a more important factor in the development of devices. Several thermal analysis techniques, such as scanning thermal microscopy [1], micro-Raman thermography [2], and infrared micro-thermography [3], have been developed to investigate thermal properties in micro- and nano-scale devices. In particular, thermoreflectance microscopy (TRM) is a contactless optical imaging technique that provides a two-dimensional thermal image of micro-electronic devices with high spatial and thermal resolution [4 -10]. In this presentation, we demonstrate a thermal imaging microscope system and their several applications, such as quantitative measurement of the surface temperature distribution of semiconductor devices, hot spot detection for failure analysis of semiconductor integrated circuits, and detection of defects, which act as laser-induced damage precursors, in high power laser optical materials.

Figure 1 shows a schematic of the thermoreflectance microscope. To build up the system, we simply combined three main components: a microscope unit, a tunable optical filter, and a dispersive spectrometer. Two CCD cameras, which allowed us to acquire the thermoreflectance spectrum and the thermoreflectance image, were attached into the system with an objective lens and a white light source. For system control, the data acquisition (DAQ) board generates trigger signals for both the camera image acquisition and the device bias modulation, and also performs precise synchronization between the two triggers. The control and signal process to obtain thermoreflectance image are described in the reference [10].

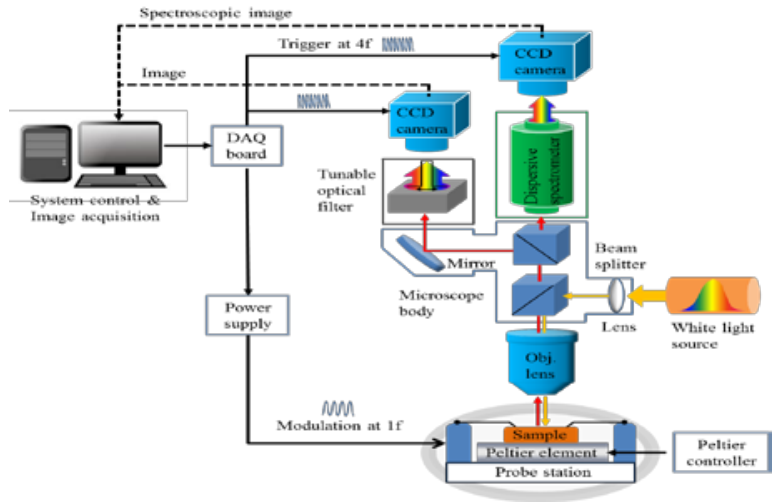

Fig. 1. Schematic of a thermoreflectance microscope system

Self-heating effect is recently considered to play an essential role in the degradation of amorphous oxide thin film transistors (TFTs). Previous thermal analysis on amorphous oxide TFTs based on conventional infrared micro-thermography, however, had limitations in studying short-channel TFTs due to its low spatial-resolution. We investigated self-heating effect of short-channel amorphous In-Ga-Zn-O TFTs with various channel length by using high resolution thermoreflectance microscopy. For the TFT with a channel length of $15 \mathrm{um}$, the device temperature due to self-heating reached $70-80{ }^{\circ} \mathrm{C}$, and local heating arose close to the center of the channel as shown in figure 2(b). The channel length dependence of thermal distribution revealed that the asymmetry of local heating weakened with the decreasing channel length due to a heat dissipation by the source and drain electrodes [7]. 
Thermal analysis of small defects in microelectronic devices becomes essential for understanding the influence of hotspots, which affect the device performance. By using TRM, submicron scale defects in micro-resistor device that are invisible in conventional optical microscope images could be clearly distinguished and individually isolated as shown in figure 2(d). The TRM can be a useful noncontact tool for both thermal characterization and submicron-scale defect isolation for a wide range of active microelectronic devices because of its high thermal and spatial resolution [8].
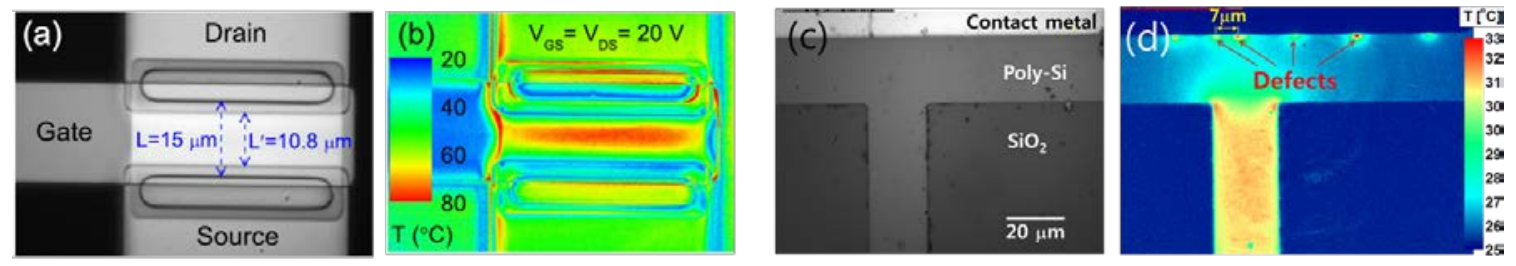

Fig.2. IGZO short channel TFT (a) Optical microscope image (b) Temperature image;

Poly-Si micro-resistor (c) Optical microscope image (d) Temperature image

Thermal characterization of individual pixels in microbolometer infrared image sensors is needed for optimal design and improved performance. In this work, we used thermoreflectance microscopy on uncooled microbolometer image sensors to investigate the thermal characteristics of individual pixels. Two types of microbolometer image sensors with a shared-anchor structure were fabricated and thermally characterized at various biases and vacuum levels by measuring the temperature distribution on the surface of the microbolometers. The results show that thermoreflectance microscopy can be a useful thermal characterization tool for microbolometer image sensors. [9].
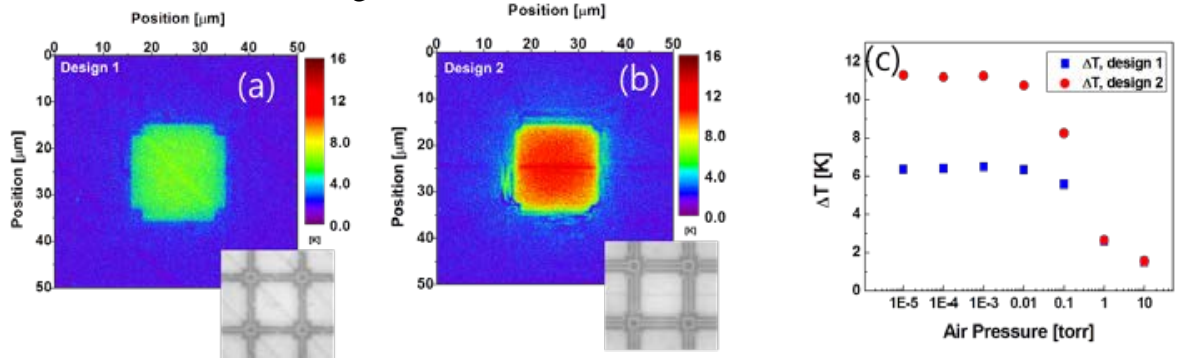

Fig. 3. Thermal distribution images of microbolometer (a) design1 and (b) design2 measured by TRM system at 1 V bias. (c) Average temperature variation of microbolometer design 1 and design 2 as a function of pressure in vacuum package

\section{ACKNOWLEDGMENT}

This work was supported by Korea Basic Science Institute grant (D37615) .

\section{REFERENCES}

[1] I. Jo et al., "Low-Frequency Acoustic Phonon Temperature Distribution in Electrically Biased Graphene”, Nano Lett. 11, 85-90, 2011

[2] D. Chae et al., "Hot Phonons in an Electrically Biased Graphene Constriction”, Nano Lett. 10, 466-471, 2009

[3] K. S. Chang et al., "Precise Temperature Mapping of GaN-Based LEDs by Quantitative Infrared Micro-Thermography”, Sensors 12, 46484660, 2012

[4] M. Farzaneh et al., “CCD-based thermoreflectance microscopy: principles and applications”, J. Phys. D: Appl. Phys. 42, 143001, 2009

[5] W. J. Choi et al., "High-speed thermoreflectance microscopy using charge-coupled device-based Fourier-domain filtering”, Opt. Lett. 38, 3581-3584, 2013

[6] W. J. Choi et al., "Fast mapping of absorbing defects in optical materials by full-field photothermal reflectance microscopy”, Opt. Lett. 38, 4907-4910, 2013

[7] J. I. Kim et al., "Thermoreflectance microscopy analysis on self-heating effect of short-channel amorphous In-Ga-Zn-O thin film transistors”, Appl. Phys. Lett. 105, 043501, 2014

[8] S. Y. Ryu et al., “ Surface Temperature Measurement and Submicron Defect Isolation for Microelectronic Devices Using Thermoreflectance Microscopy”, Int. J. Thermophys, 36, 1217-1225, 2015

[9] S. Y. Ryu et al., "Thermal Characterization of Individual Pixels in Microbolometer Image Sensors by Thermoreflectance Microscopy", Journal of Semiconductor Technology and Science, 15, 533-538, 2015

[10] D. U. Kim et al., "Quantitative temperature measurement of multi-layered semiconductor devices using spectroscopic thermoreflectance microscopy”, Optics Express, 24, 13906-13916, 2016. 